\title{
LA MALNUTRICIÓN COMO FACTOR PRINCIPAL EN LA DIABETES MELLITUS NO DEPENDIENTE EN ADULTOS DE LOS MERCADILLOS EN TACNA
}

\author{
Soledad Bornás Acostal, Guillermo Bornaz Acosta ${ }^{I}$ \\ Vicente Chambilla Quispe
}

\section{RESUMEN}

Se realizó una campaña de despistaje de DIABETES en los socios de los mercadillos de la ciudad de Tacna. El despistaje consistió en una determinación de glicemia por un método enzimático y glucotest. En la muestra de socios, el $54.55 \%$ presentan obesidad moderada y presentan diabetes tipo II solo los mayores de 40 años en un porcentaje alrededor del $4 \%$ comprobandose que la obesidad adquirida por malnutrición fue el factor desencadenante de este tipo de diabetes.

\section{A BSTR ACT}

A campaign of despistaje of DIABETES in the partners of the mercadillos of the city of Tacna was done. The despistaje consisted on a glicemia determination for an enzymatic method and glucotest. In the sample of partners participants, $54.55 \%$ presents moderate obesity and they have diabetes type II alone those bigger than 40 years in a percentage around $4 \%$ being proven that the obesity acquired by malnutrition was the factor desencadenante of this diabetes type.

\section{INTRODUCCIÓN}

La diabetes mellitus se constituye actualmente en un problema de salud pública en todo el mundo y está considerada como una de las enfermedades crónicas que ha tenido una gran emergencia en la última década, al extremo de ser caracterizada como una epidemia. Datos de la Organización Mundial de la Salud (WHO) y de la Federación Internacional de Diabetes (IDF) para el año 1994, confirman la existencia de aproximadamente 110 millones de pacientes diabéticos con una franca tendencia a llegar a los 240 millones para el año 2010.

A nivel regional, Latinoamérica está considerada como una de las poblaciones que doblará el número de pacientes diabéticos de 12 millones a 24 millones en el 2010, tan igual como en otras regiones en vías de desarrollo producto del fenómeno de envejecimiento poblacional y de estilo de vida nocivos.

En nuestro pais, estudios epidemiológicos realizados recientemente permiten asumir la existencia aproximada de un millón de pacientes diabéticos, de los cuales menos de la mitad están en posibilidades de ser diagnosticados en las actuales condiciones. Este panorama epidemiológico está produciendo un alto costo económico, social y familiar, producto de la demanda de atención medica que ocasiona su diagnóstico, tratamiento y control; situación que se ve empeorada por el bajo nivel

Biologos. 
educativo de la población afectada y la inadecuada capacitación técnica de los profesionales que asisten a dichos pacientes, lo que explica la frecuente presentación clínica de complicaciones agudas y crónicas como la insuficiencia renal, retinopatía, neuropatía, enfermedad coronaria y cerebrovascular.

Sin intervención de las hormonas glucagon/ insulina, la concentración de glucosa en la sangre presentaría una fluctuación grande a lo largo de la jornada que pondría en riesgo la vida de nuestro organismo, pero gracias a estas señales se evitan estas fluctuaciones peligrosas.

La diabetes de tipo II es la predominante dentro de nuestras poblaciones, también conocida como de inicio maduro. Tiene como característica intolerancia a la glucosa generada por factores genéticos y ambientales. Varía desde estados donde predomina la resistencia a la insulina con relativa deficiencia de insulina a estados de deficiencia de insulina; secretora predomina sobre la resistencia.

En el conjunto de acciones adoptadas para evitar su aparición o progreso, están las destinadas a modificar el estilo de vida y las características socioambientales que, junto con los factores genéticos, constituyen causas desencadenantes de la enfermedad.

Existen factores de riesgo para la diabetes tipo II que son modificables: obesidad, sedentarismo, hiperlipidemas, hipertensión, tabaquismo y nutrición inapropiada. La población en riesgo está constituida por mayores de 40 años, obesos, con antecedentes de diabetes mellitus en familiares, mujeres con hijos macrosómicos $\mathrm{y} / \mathrm{o}$ antecedentes obstétricos patológicos, menores de 40 años con enfermedad coronaria, hipertensos, hiperlipidémicos.

La dieta es uno de los pilares fundamentales de la prevención y tratamiento de la diabetes.

El presente trabajo tiene como finalidad establecer que una inadecuada nutrición es uno de los principales factores de diabetes meilitus tipo II en la población adulta mayor de 40 años que trabaja en los diferentes mercadillos de la ciudad de Tacna. Consiste en una dieta hipercalórica con exceso de carbohidratos y grasas.

\section{MATERIAL Y MÉTODOS}

Durante el año de 1999, se tomaron muestras de sangre de 180 adultos obesos que trabajan en los mercadillos de la Ciudad de Tacna, con lo cual se llevaron a cabo las siguientes determinaciones:

\subsection{GLICEMIA ENZIMÁTICA}

Se llevó a cabo la determinación de glucosa en sangre utilizando el método enzimático.

\section{Fundamentos del Método}

El esquema de reacción fue el siguiente:

glu $\cos a+\mathrm{O}_{2}+\mathrm{H}_{2} \mathrm{O} \longrightarrow \cdots \rightarrow$ ácidoglucónico $+\mathrm{H}_{2} \mathrm{O}_{2}$

$2 \mathrm{H}_{2} \mathrm{O}_{2}+4-\mathrm{AF}+$ fenol $----\rightarrow$ quinonacoloreada $+4 \mathrm{H}_{2} \mathrm{O}$ GOD

\section{Reactivos Provistos POD}

Standard

Solución de glucosa $1 \mathrm{~g} / \mathrm{l}$.

GOD/POD : Solución de glucosa oxidasa $(1000 \mathrm{U} / \mathrm{ml})$ y peroxidasa $(120 \mathrm{U} /$ $\mathrm{ml})$.

Reactivo 4-AF : Solución de 4-aminofenazona 25 $\mathrm{mmol} / \mathrm{l}$ en Buffer -Tris $0,92 \mathrm{~mol} /$ I.

Reactivo fenol : Solución de fenol $55 \mathrm{mmol} / \mathrm{l}$.

\section{Muestra}

Suero o plasma

a) Recolección: se obtuvo el suero o plasma de la manera usual.

\section{Procedimiento}

En tres tubos de fotocolorimetro marcados $B$ (Blanco) S (Standard) y D(Desconocido) se colocó:

\begin{tabular}{|c|c|c|c|}
\hline & B & S & D \\
\hline Standard & - & $20 \mathrm{ul}$ & - \\
\hline Muestra & - & - & $20 \mathrm{ul}$ \\
\hline Reactivo de Trabajo & $2 \mathrm{ml}$ & $2 \mathrm{ml}$ & $2 \mathrm{ml}$ \\
\hline
\end{tabular}


Se incubó 10 minutos en baño de agua a $37^{\circ} \mathrm{C}$. Luego se leyó en un espectrofotómetro a $505 \mathrm{~nm}$ o en fotocolorímetro con filtro verde $(490-530 \mathrm{~nm})$ llevando el aparato a cero con el blanco.

\section{Estabilidad de la mezcla de reacción final}

El color de reacción final fue estable 1 hora, por lo que la absorbencia debió ser leída dentro de este lapso.

\section{Cálculo de los resultados}

$$
g \text { de glucosa } / l=D x f
$$

donde:

$$
f=\frac{1,00 g / l}{S}
$$

\section{Valores de referencia}

Suero o plasma: $0,70-1,10 \mathrm{~g} / \mathrm{l}$

\subsection{GLUKOTEST}

Se llevó a cabo la determinación de la glicemia por el método del GLUKOTEST.

\section{Preparación y realización de la medición}

Seguir las instrucciones de empleo detalladas de los fotómetros de reflexión mencionados.

Codificación: Al abrir el envase de tiras reactivas se codificó el aparato mediante la tira de código adjunta. Se tuvo en cuenta que el procedimiento de codificación de los aparatos Reflolux y Reflolux II M es diferente frente a Reflolux S. La tira de código no debe guardarse en el tubo de tiras reactivas.

Obtención de la sangre: Lavarse las manos con agua caliente y jabón, y secárselas bien. Empleando un desinfectante o no, el lugar de la punción debe estar completamente seco antes de la obtención de la sangre, ya que en otro caso se da el riesgo de resultados erróneos.

Hacer la punción con una lanceta en un lado de la yema del dedo. Secar la primera gota de sangre. Apretar ligeramente la yema del dedo para obtener una gran gota de sangre pendiente.
Aplicación de la sangre: Aplicar la gota de sangre de manera que ambas zonas de test queden completamente cubiertas. No extender la sangre. Pulsar inmediatamente la tecla TIME.

Al cabo de $60 \mathrm{seg}$. secar la gota de sangre con algodón hidrófilo limpio (no emplear celulosa), ejerciendo una moderada presión. Volver a secar dos veces con una cara limpia del algodón.

Indicación del valor medido: Después del secado, introducir la tira reactiva hasta el tope en la entrada de tiras reactivas, con la zona de test dirigida hacia la tecla ON/OFF.

A los 120 segundos, el resultado es indicado automáticamente.

Comparación visual: Para controlar la plausibilidad del resultado se recomienda comparar visualmente, después de la medición, con los colores indicados en la etiqueta del tubo de tiras reactivas. La zona de test está compuesta de dos áreas con distinta sensibilidad para glucosa. El área inferior (cerca del mango) permite una buena diferenciación en el intervalo entre 20 y $120 \mathrm{mg} / \mathrm{dl}(1-7 \mathrm{mmol} / \mathrm{l})$ y el área superior en el intervalo superior a $120 \mathrm{mg} / \mathrm{dl}$ (7 mmol/l).

En caso de que, después de 2 minutos, el valor de glucemia sobrepase $300 \mathrm{mg} / \mathrm{dl}(17 \mathrm{mmol} / \mathrm{l})$, repetir la comparación de colores después de otro minuto, es decir 3 minutos en total después de la aplicación de la gota.

Los colores de comparación están impresos específicamente para cada lote de tiras reactivas (diferentes números de código), por ello no emplear la escala cromática de otro estuche.

\section{Dietética}

Se determinaron los hábitos nutricionales de la muestra de trabajadores de los mercadillos, utilizando una encuesta dietetica.

\section{RESULTADOS}

Durante el año de 1999, se realizó en los diferentes mercadillo una campaña de despistaje, asistiendo una buena cantidad de socios trabajadores (180), a los cuales en ayunas se les determinó su glicemia, con la finalidad de obtener valores basales de glucosa y detectar si alguno de ellos presenta 
HIPERGLICEMIA, principal signo clínico del padecimiento de DIABETES.

Los individuos que se sometieron al análisis presentaron edades de 21 a 80 años, predominando los mayores de 40 años. El Gráfico 1 muestra la relación entre el peso, edad y la glicemia de los diferentes trabajadores; se nota claramente que conforme se incrementa la edad, tambien se aumenta el peso aproximadamente hasta los 60 años, y la glicemia lo hace hasta los 80 años.

EI Gráfico 2 nos refleja que de los trabajadores de la muestra, el $11.36 \%$ presentaron Hiperglicemia entre los 40 a 80 años, presentándose el mayor indice entre los individuos de 51 a 60 años. En relación al sexo (Gráfico 3), los trabajadores de sexo femenino presentaron hiperglicemia, del $11.36 \%$, y el $7.97 \%$ correspondieron a mujeres probablemente con padecimiento de diabetes, esto porque un mayor porcentaje de trabajadores fueron mujeres.

De la encuesta dietética, las dietas que normalmente venían consumiendo estos trabajadores basicamente eran ricas en carbohidratos y relativamente en grasas preparadas por vendedores de comida ambulantes y algunos restaurantes.

Gráfico 1. Peso (kg.) y valores de glucosa en sangre de diferentes trabajadores de los mercadillos de la ciudad de Tacna, según intervalos de edad.

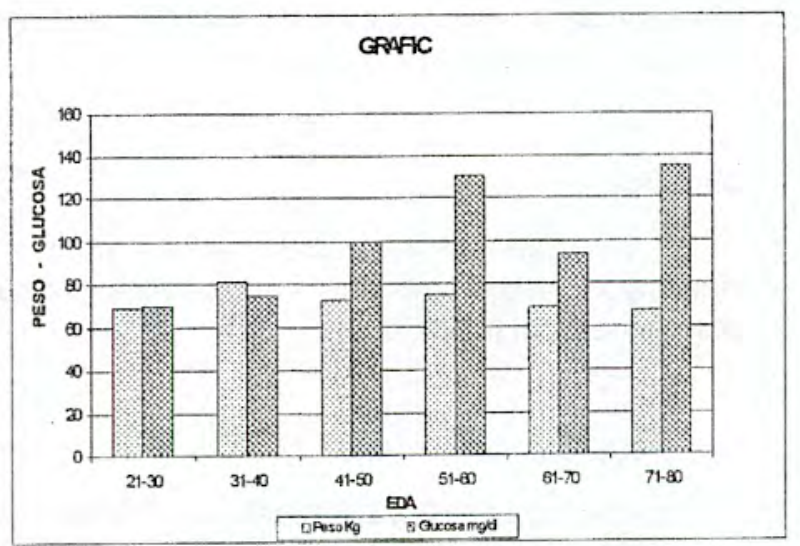

Gráfico 2. Frecuencia de Diabéticos en Socios de los Diferentes Mercadillos de Tacna.

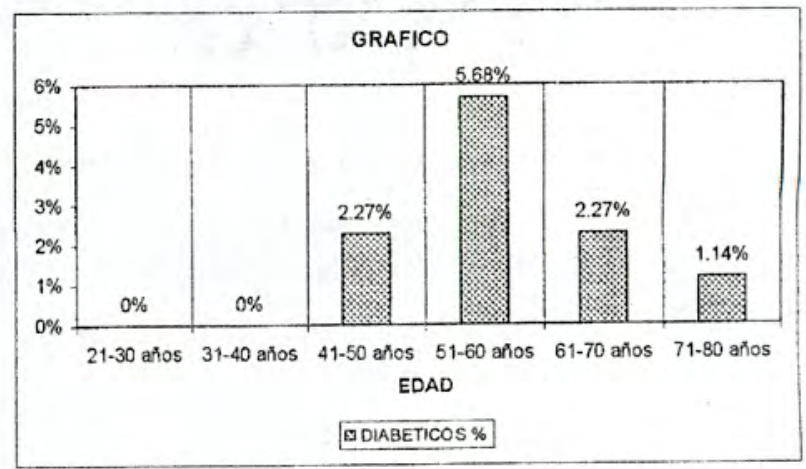

Gráfico 3. Frecuencia de Diabéticos en Socios de los Diferentes Mercadillos de Tacna.

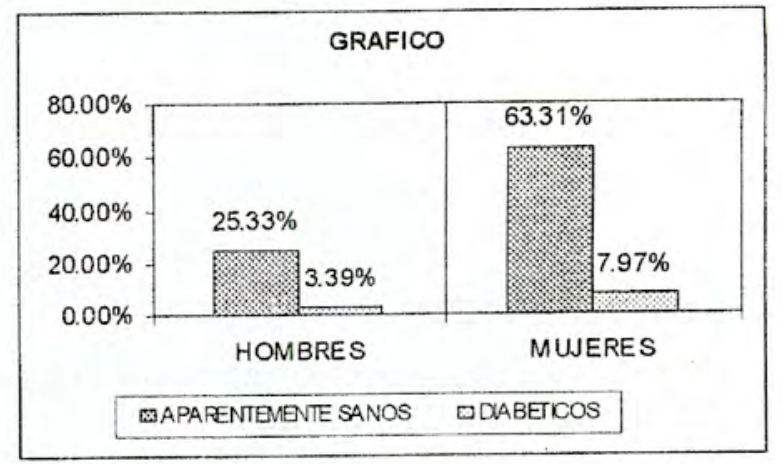

Nota: Se debe tener en cuenta que el mayor porcentaje de la población de socios que se realizó el análisis de glucosa fueron mujeres.

\section{DISCUSIÓN}

La Diabetes Mellitus INSULINO NO DEPENDIENTE, también conocida como diabetes adulto estable, corresponde al $85 \%$ de los diabéticos. Aparece generalmente después de los 45 años de edad, en forma lenta. Se diagnóstica frecuentemente por otra patología o por sus complicaciones. Los pacientes se caracterizan por tener insulina circulante en cantidad normal o aumentada, presentando en los obesos resistencia a la insulina.

Numerosas investigaciones permiten afirmar que la Diabetes Mellitus es una enfermedad en aumento creciente. Este hecho ha sido confirmado por estudios 
realizados en EE.UU. que demuestra que el número de diabéticos en la década de 1966 - 1975 ha aumentado un $50 \%$ (11). En Chile un estudio realizado en 1979 mostró una prevalencia de $5.3 \%$ en la población mayor de 20 años.

En nuestro país las campañas de despistaje que se efectúan en diferentes lugares demuestran un aumento considerable de hiperglucémicos (diabéticos), especialmente a la edad adulta.

Según nuestra investigación, los socios de los diversos mercadillos de Tacna mostraron un $11.36 \%$ de diabéticos mayores de 44 años de edad (Gráficos 2 y 3 ). Esto concuerda con las investigaciones realizadas en otros países.

La diabetes insulino no dependiente es fundamentalmente hereditaria. El factor desencadenante es la obesidad a través de la secuencia: obesidad, hiperinsulinismo, disminución del número de receptores periféricos a la hormona.

En algunos casos de los hiperglucémicos detectados en los mercadillos de Tacna, conocían su problema y ya tenían un previo tratamiento nutricional.

A cada individuo, además de efectuársele un análisis de glucosa, también se le realizó una encuesta indicando sus datos antropométricos, antecedentes familiares con diabetes y el tipo de dieta que mantiene diariamente. De esta encuesta se observó que el $54.55 \%$ presenta una obesidad moderada, y dentro de este grupo el $5 \%$ son extremadamente obesos.

Dentro del grupo de diabéticos detectado en las campañas de Despistaje de Diabetes, el $60 \%$ presentaron obesidad, con lo que se demuestra que este factor fue principal el el desencadenante de la hiperglicemia que conduce a que el paciente presente Diabetes Mellitus Tipo II.
En cuanto al sexo no se puede considerar como un causal de la Diabetes Mellitus. A pesar de que las mujeres tienden a engordar con mayor facilidad que los hombres por el acúmulo de tejido adiposo, no se ha visto ninguna diferencia. Por otro lado, la gran mayoría de personas que se hicieron el análisis fueron mujeres y muy poco varones que tienen una vida sedentaria.

En cuanto a la alimentación de estos individuos, especialmente en el caso de los obesos, está constituida mayormente por grasas, carbohidratos (harinas, pastas, papa, azúcar, dulces, etc.), muy poco carnes, verduras, frutas, etc. También cabe recalcar que consumen sus alimentos en cantidad y no en calidad, y eso se debe a que la mayoría consiguen sus menús de restaurantes o centros de comida que no tienen un criterio gastronómico adecuadamente nutricional para sus comensales, simplemente sus actividades tienen un fin lucrativo.

Se aplicó un Plan Nutricional a cada hiperglucémico detectado, siguiendo con la Dietoterapia descrita en la parte de material y métodos. Ninguno de estos individuos estuvo en tratamientos con medicinas por otro tipo de patología.

\section{CONCLUSIONES}

1. La Diabetes Mellitus no insulino dependiente es la más frecuente y se presenta generalmente después de los 40 años en los Socios de los diferentes Mercadillos de la Ciudad de Tacna.

2. El factor desencadenante de la Diabetes Mellitus tipo II fué la obesidad obtenida por un excesivo consumo de Carbohidratos y grasas.

3. En socios menores de 40 años no se presentó ningún caso de diabetes. 


\section{REFERENCIAS BIBLIOGRÁFICAS}

ALBRINK, M.J.; DAVIDSON, P.C.; y NEUMANN, R. :LIprd lowering effect of high - carbohidrate, highfiber. Diabetes; 35: 324, 1989.

BRUNZELL, J.D.; LERNER, R.L.; PORTE, D. y BIERMAN, E.L.: Effect of a fat-free high - carbohidrate diet on diabetic subjects with fasting hyperglicemia Diabetes, 23:138; 1994.

DUNN, F. :Treatment of Lipid Disorders in Diabetes Mellitus Med. Cli N.A. 1988; 72:1379-1394.

FOSTER, D.W. : From Glycogen to Ketones - and back Diabetes 33:1188-1199. 1984.

HOLVEY, SM. : The Diabetes education team in the management of non insulin - dependent diabetes mellitus. Metabolismo 1987.

Mc GARRY, J.D. y Col: From Dietary glucose o liver glycogen. The full circle round. Ann. Rev. Nutrition 7:51-73,1998.

MONTGOMEKY, R. ph D. y Col.: Bioquimica, casos y texto. 6ta. edición, Editorial Horcourt Brace, de España S.A. 1998.
ORGANIZACIÓN PANAMERICANA DE LA SALUD OPS $\theta$ Instituto Internacional de Ciencias de la Vida ILSI-North América. Conocimiento actuales sobre nutrición. 6ta. Edición. Publicación Científica Washington D.C. E.U.A.1991.

OLIVARES, S. y Col:: Nutrición, prevención de riesgos y tratamiento dietético. Confederación Latinoamericana de Nutricionistas. Dietistas. 2da. Edición. Santiago de Chile, 1996.

RUIZ, M. y Col. Ateroesclerosis y medidas higiénicodietéticas en: Cordova R. Col Ateroesclerosis al Dia Venezuela, Ediciones Lerner Ltda.1987.

TECHNICAL REPORT SERIES EXPERT: Comité en Diabetes Mellitus N648. 1980.

TROWELL, H.C.: Dietary - fiber hypothesis of the etiology of diabetes mellitus. Diabetes, 24:762, 1995.

TRUGLIA, J. y Col.: Insulin Resistanse: Receptor and post binding defect in Human obesity and non insulin dependent diabetes mellitus. Ann J. med. 79 (Suppl.2B) 13, 1985 . 